\title{
Gastrointestinal obstruction associated with Chlamydia trachomatis
}

\author{
D J Pegg, A W M C Owen
}

\begin{abstract}
A patient is reported in whom adhesive obstruction of the small bowel was due to generalised peritonitis caused by Chlamydia trachomatis. It is concluded that any sexually active female with unexplained peritonitis or adhesive obstruction of the small bowel should be screened for chlamydial infection.
\end{abstract}

Despite the prevalence of infection of the genital tract and pelvic inflammatory disease caused by Chlamydia trachomatis, generalised abdominal manifestations of chlamydial infection are unusual.

\section{Case report}

A female aged 18 years was admitted to hospital on three separate occasions in 3 weeks because of repeated attacks of abdominal pain and vomiting. Observation and appropriate investigations excluded pregnancy, appendicitis and apparently infection of the genital tract. During her third admission laparotomy was performed for persistent colicky abdominal pain accompanied by clinical and radiological signs of gastrointestinal obstruction. The peritoneal cavity contained $900 \mathrm{ml}$ of straw coloured fluid and there was a generalised serositis of the infracolic compartment. The liver was normal and apart from inflammation of their covering serosa, the appendix, uterus and fallopian tubes appeared normal. There were widespread tenacious adhesions between adjacent loops of small bowel with adhesive obstruction of the mid ileum. Peritoneal fluid was submitted for routine bacterial culture whilst swabs from the fallopian tubes and cervix were sent for routine culture and the isolation of chlamydia. The obstruction was relieved, appendicectomy was performed and the peritoneal cavity was lavaged with a solution of tetracycline ( $1 \mathrm{gm} / \mathrm{l})$.

No pathogens were grown on routine culture and

University Department of Surgery, University Hospital of South Manchester, Manchester, UK

DJ Pegg, A W M C Owen unfortunately the swabs for chlamydial culture were inadvertently sent in virus transport medium, culture was not attempted. However, the swabs from the fallopian tubes and cervix were strongly positive for chlamydia when tested by ELISA (IDEIA Mark 2 Boots-Celltech and IDEIA Mark 3 (Novo Biolabs Ltd). In the absence of Staphylococcus aureus they were regarded as true positives. Samples of blood taken on two separate occasions showed a chlamydial antibody titre of $1 / 8192$ using a single serotype MIF test. Chlamydial IgM antibody was detected in one of these samples at the screening dilution of $1 / 8$.

Following surgery the patient was treated with doxycycline $100 \mathrm{mg}$ daily for 4 weeks and her symptoms resolved completely. Although her sexual partner was asymptomatic, chlamydia was isolated from his urethra and he was treated with oxytetracycline $500 \mathrm{mg}$ bd for 2 weeks.

\section{Discussion}

In 1986 there were 157,000 new cases in the UK of non-specific infection of the genital tract of which 51,500 occurred in women ${ }^{1}$ and it has been suggested that approximately half of these may be due to Chlamydia trachomatis. ${ }^{2}$ Despite the prevalence of sexually transmitted infection and pelvic inflammatory disease caused by chlamydiae, more widespread abdominal manifestations are unusual. The best known is the Fitz-Hugh-Curtis syndrome, peritonitis in the right upper quadrant and perihepatitis associated with infection of the genital tract. This produces characteristic "violin-string" adhesions between the capsule of the liver and the adjacent abdominal wall. ${ }^{3}$ Originally it was thought this syndrome was caused by Neisseria gonorrhoeae ${ }^{4}$ but Chlamydia trachomatis has also been implicated. ${ }^{5}$ Neither perihepatitis, salpingitis or pelvic inflammatory disease are invariably present. ${ }^{5}$ There have been two reports of generalised peritonitis caused by chlamydiae. ${ }^{56}$ The detection of chlamydial antigen in the fallopian tubes and the high titres of antichlamydial antibody noted in our patient are highly suggestive that the peritonitis was caused by chlamydia. $^{7}$

We believe this is the first report of adhesive obstruction of the small bowel caused by chlamydial 
peritonitis. It is possible that some previously reported cases of so called "primary" peritonitis in young women may have been unrecognised chlamydial infection. We recommend that any sexually active female with unexplained peritonitis or adhesive obstruction of the small bowel should be screened for chlamydial infection.

We thank Mr E N Gleave for permission to publish this case and wish to acknowledge the help and advice of Dr M E Macaulay and staff of the Central Serology Laboratory, University Hospital of South Manchester.

Address for reprints: A W M C Owen, Dept of Surgery, University Hospital of South Manchester, Nell Lane, Manchester M20 8LR, UK
1 New cases seen at NHS genito-urinary medicine clinics 1976-1986. Statistical bulletin. London. Department of Health and Social Security 1988.

2 Communicable disease surveillance centre. Sexually transmitted diseases surveillance in Britain 1984. Br Med J 1986;293: 942-3.

3 Curtis AH. A cause of adhesions in the right upper quadrant. JAMA 1930;98:1221-2.

4 Fitz-Hugh T. Acute gonococcal peritonitis in the right upper quadrant in women. JAMA 1934;102:2094-6.

5 Muller-Schoop JW, Wang SP, Muzinher J, Schlapfer HU, Knoblaugh M, Amman RW. Chlamydia trachomatis as possible cause of peritonitis and perihepatitis in young women. $\mathrm{Br}$ Med J 1978;1:1022-4.

6 Duffy S, Cawdell G, Fieldman N. Unusual presentation of chlamydial peritonitis: case report. Genitourin Med 1985; 61:202-3.

7 Treharne JD, Ripa KT, Mardh PA, Sevensson L, Westrom L, Darougar S. Antibodies to Chlamydia trachomatis in acute salpingitis. Br J Venereol Dis 1979;55:26-9.

Accepted for publication 28 September 1989 